\title{
Expression Pattern Suggests a Role of MiR399 in the Regulation of the Cellular Response to Local Pi Increase During Arbuscular Mycorrhizal Symbiosis
}

\author{
Anja Branscheid, ${ }^{1}$ Daniela Sieh, ${ }^{1}$ Bikram Datt Pant, ${ }^{1}$ Patrick May, ${ }^{1}$ Emanuel A. Devers, ${ }^{1}$ Anders Elkrog, ${ }^{2}$ \\ Leif Schauser, ${ }^{2}$ Wolf-Rüdiger Scheible, ${ }^{1}$ and Franziska Krajinski ${ }^{1}$ \\ ${ }^{1}$ Max Planck Institute of Molecular Plant Physiology, Science Park Golm, 14476 Potsdam, Germany; ${ }^{2}$ Bioinformatics \\ Research Center, Aarhus University, 8000 Aarhus, Denmark
}

Submitted 25 November 2009. Accepted 17 March 2010.

\begin{abstract}
Many plants improve their phosphate $(\mathrm{Pi})$ availability by forming mutualistic associations with arbuscular mycorrhizal (AM) fungi. Pi-repleted plants are much less colonized by AM fungi than Pi-depleted plants. This indicates a link between plant $\mathrm{Pi}$ signaling and $\mathrm{AM}$ development. MicroRNAs (miR) of the 399 family are systemic Pi-starvation signals important for maintenance of Pi homeostasis in Arabidopsis thaliana and might also qualify as signals regulating $\mathrm{AM}$ development in response to $\mathrm{Pi}$ availability. MiR399 could either represent the systemic low-Pi signal promoting or required for $\mathrm{AM}$ formation or they could act as counter players of systemic Pi-availability signals that suppress AM symbiosis. To test either of these assumptions, we analyzed the miR399 family in the AM-capable plant model Medicago truncatula and could experimentally confirm 10 novel MIR399 genes in this species. Pi-depleted plants showed increased expression of mature miR399 and multiple pri-miR399, and unexpectedly, levels of five of the 15 pri-miR399 species were higher in leaves of mycorrhizal plants than in leaves of nonmycorrhizal plants. Compared with nonmycorrhizal $\mathrm{Pi}$-depleted roots, mycorrhizal roots of Pi-depleted $M$. truncatula and tobacco plants had increased $P i$ contents due to symbiotic Pi uptake but displayed higher mature miR399 levels. Expression levels of MtPho2 remained low and PHO2-dependent Pi-stress marker transcript levels remained high in these mycorrhizal roots. Hence, an AM symbiosis-related signal appears to increase miR399 expression and decrease $\mathrm{PHO2}$ activity. MiR399 overexpression in tobacco suggested that miR399 alone is not sufficient to improve mycorrhizal colonization supporting the assumption that, in mycorrhizal roots, increased miR399 are necessary to keep the MtPho2 expression and activity low, which would otherwise increase in response to symbiotic Pi uptake.
\end{abstract}

Phosphorus (P) is second to nitrogen as the most-limiting element for plant growth. $\mathrm{P}$ not only is a major component of fundamental macromolecules, it also plays an important role in

Corresponding author: Franziska Krajinski; Telephone: +49-331-5678355; E-mail: krajinski@mpimp-golm.mpg.de

* The $\boldsymbol{e}$-Xtra logo stands for "electronic extra" and indicates that three supplementary tables and two supplementary figures published online. energy transfer, regulating enzymatic reactions, and different metabolic pathways. $\mathrm{P}$ is taken up by plant roots as inorganic phosphate $(\mathrm{Pi})$, which is one of the least plant-available nutrients in the soil. To maintain optimal yield, a majority of agricultural systems depend on the application of $\mathrm{P}$ fertilizers. Their energy-intensive production depends on nonrenewable resources, which could be depleted by as early as 2060 (Vance et al. 2003).

Owing to the poor soil availability of $\mathrm{Pi}$, plants have evolved a variety of adaptive strategies to improve $\mathrm{Pi}$ acquisition. These include altered root morphology, exudation of organic acids, phosphatases, and nucleases in order to solubilize inorganically and organically bound $\mathrm{Pi}$ and the establishment of a symbiotic relationship with arbuscular mycorrhizal (AM) fungi (Harrison 1999; Hause and Fester 2005; Parniske 2008). AM fungi are present in nearly all terrestrial ecosystems, forming mutualistic symbiosis with the roots of approximately $80 \%$ of vascular plants. They often improve Pi uptake and plant growth. The ability of AM fungal hyphae to grow beyond the root's Pi-depletion zone and deliver $\mathrm{Pi}$ to the host plant is thought to be the main basis for their positive effects on Pi uptake (Smith and Read 1997). The establishment of an AM symbiosis requires a complex developmental program of both plant and AM fungus. During the last years, plant mutants impaired at different steps of the AM colonization process have been identified and revealed insight into the plant developmental program and cell reprogramming during AM symbiosis (Parniske 2008). Additional insight in the analysis of molecular events during AM symbiosis has been achieved recently by the identification of plant Pi transporters that are either specifically induced in response to AM symbiosis (Glassop et al. 2005; Harrison et al. 2002; Nagy et al. 2005; Paszkowski et al. 2002) or strongly upregulated in mycorrhizal roots but having a basal expression in nonmycorrhizal roots (Chen et al. 2007; Maeda et al. 2006; Rausch et al. 2001). This induction of specific Pi transporters in mycorrhizal roots and the parallel downregulation of conventional $\mathrm{Pi}$-uptake systems suggests that, in mycorrhizal roots, two Pi-uptake pathways are present: the direct Pi-uptake pathway involving Pi transporters expressed in the epidermis and in root hairs, and a mycorrhizal uptake pathway that involves the uptake of Pi from the soil by AM fungal transporters located in extraradical fungal hyphae and a subsequent transport to the intraradical hyphae in which it is provided to the plant (Bucher 2007). Interestingly, components of the direct Pi-uptake pathway are often downregulated in response 
to AM symbiosis leading to a reorganization of Pi uptake (Bucher 2007; Smith et al. 2003). This is intriguing because other P-dependent regulatory mechanisms seem to operate in the other direction, i.e., a high $\mathrm{Pi}$ status of the plant restricts AM symbiosis development. Recently, it was reported that lyso-phosphatidylcholine (LPC) acts as a mycorrhizal signal by inducing the mycorrhiza-specific StPt3 promoter in potato roots (Drissner et al. 2007). However, a high-Pi signal is epistatic to this LPC signal, making Pi-repleted roots LPC insensitive, and the expression of StPt3 and mycorrhizal Pi uptake is repressed in Pi-repleted roots (Nagy et al. 2008).

Knowledge about how plants are able to transfer Pi-starvation signals and maintain Pi homeostasis has been obtained recently by identifying components of Pi-starvation signaling in Arabidopsis (Aung et al. 2006; Bari et al. 2006; Chiou et al. 2006; Franco-Zorrilla et al. 2007; Pant et al. 2008). The MYB transcription factor PHR1 plays a central role in signaling of primary Pi responses (Rubio et al. 2001). This is emphasized by the fact that approximately $90 \%$ of a set of $64 \mathrm{Pi}$ starvation-inducible transcripts show attenuated induction in Arabidopsis phrl mutants (Bari et al. 2006), demonstrating the central importance of PHR1 in the Pi-starvation response. PHR1
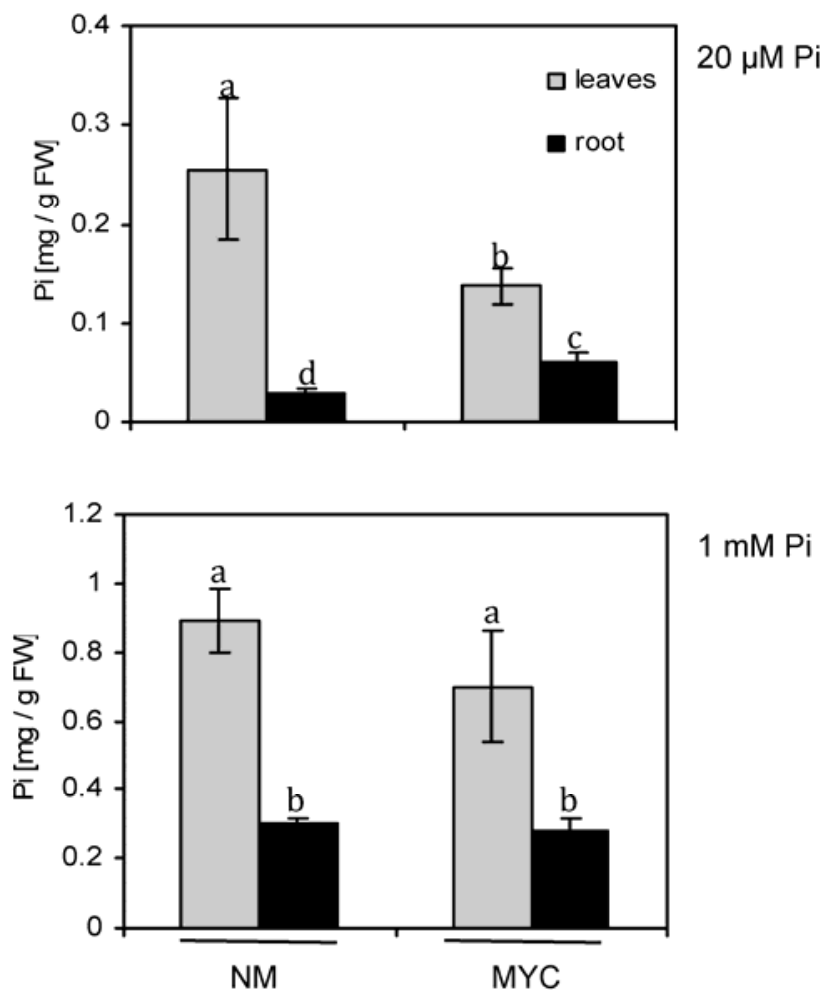

Fig. 1. Soluble phosphate $(\mathrm{Pi})$ concentrations in Pi-repleted $(1 \mathrm{mM})$ and Pi-depleted $(20 \mu \mathrm{M})$ leaves and roots of mycorrhizal (MYC) and nonmycorrhizal (NM) Medicago truncatula plants. Data represent the mean \pm standard deviation of five independent replicates containing two plants each. Values labeled with the same letter are not significantly different. binds to a cis element with an imperfect palindromic sequence (GNATATNC) (Rubio et al. 2001) and induces the expression of many Pi starvation-inducible genes, including microRNAs (miR) of the 399 family (Bari et al. 2006). Members of this miR family are able to bind and cleave the $\mathrm{PHO} 2$ transcript (Allen et al. 2005) or to cause translational repression of PHO2 (Bari et al. 2006; Chiou et al. 2006; Pant et al. 2009). The ubiquitin-conjugating enzyme $\mathrm{PHO} 2$ negatively regulates the expression of a subset of Pi starvation-inducible genes, including several Phtl family Pi transporter genes (Aung et al. 2006; Bari et al. 2006; Chiou et al. 2006). Hence, miR399 can be regarded as Pi-starvation signals leading to $\mathrm{PHO} 2$ suppression and thus derepressing the Pi-starvation response and $\mathrm{Pi}$ uptake capacity. MiR399 activity can be quenched by noncoding RNA IPS1 through a novel mechanism termed target mimicry (Franco-Zorrilla et al. 2007). MiR399 also serve as phloem-mobile long-distance signals communicating Pi status between shoots and roots (Lin et al. 2008; Pant et al. 2008).

An important aim of this work was to investigate a potential role of miR399 as regulators in the establishment of AM symbiosis that occurs in Pi-depleted AM-capable plants, such as Medicago truncatula or tobacco (Nicotiana tabacum). For this purpose, miR399-overexpressing tobacco plants were characterized concerning their mycorrhizal phenotype under lowand high-Pi conditions. In addition, we characterized the miR399 family in the model legume $M$. truncatula. Analysis of miR399 primary transcripts and mature miR399 levels suggest the existence of a mycorrhiza-induced signal leading to increased miR399 transcription in leaves and to increased levels of mature miR399 in mycorrhizal roots.

\section{RESULTS}

\section{AM symbiosis development is suppressed by $\mathbf{P i}$ in Medicago truncatula.}

Since miR399 represent a systemic Pi-starvation signal, it can be speculated that miR399 is the systemic signal leading to enhanced AM formation in Pi-depleted plants. In order to study the regulation of miR399 during AM symbiosis, M. truncatula plants were grown with or without Glomus intraradices inoculation and were fertilized with either $20 \mu \mathrm{M}$ or $1 \mathrm{mM}$ Pi. These two Pi concentrations were selected after an initial experiment in which $M$. truncatula plants were fertilized with 0 , $0.02,1$, or $2 \mathrm{mM} \mathrm{Pi}$ and were grown for 5 weeks. After 5 weeks, Pi concentrations in leaves did not differ significantly between the $0-\mathrm{mM}$ and the $20-\mu \mathrm{M}$ treatments or between the 1- and 2-mM treatments (data not shown). Hence, we selected $20 \mu \mathrm{M}$ and $1 \mathrm{mM}$ as contrasting Pi fertilization treatments in our experiment. All experiments were carried out using at least five independent replicates. All plants were harvested after 5 weeks. The resulting phenotype of the plants is shown in Supplementary Figure S1. Pi contents of roots and shoots were measured. Plants that were fertilized with $1 \mathrm{mM}$ Pi showed clearly increased Pi concentrations in both roots and shoots (Fig. 1). Mycorrhizal plants at low-Pi conditions had increased root $\mathrm{Pi}$ concentrations. This is most likely a consequence of

Table 1. Mycorrhizal parameters of Medicago truncatula roots $^{\mathrm{a}}$

\begin{tabular}{llccccc}
\hline Myc & Pi condition & F $(\%)$ & M $(\boldsymbol{\%}) \pm$ SD & m $(\boldsymbol{\%}) \pm$ SD & A $(\boldsymbol{\%}) \pm$ SD & a $(\%) \pm$ SD \\
\hline- & $1 \mathrm{mM}$ or $20 \mu \mathrm{M} \mathrm{Pi}$ & 0 & 0 & 0 & 0 & 0 \\
+ & $1 \mathrm{mM} \mathrm{Pi}$ & 100 & $57.59 \pm 7.95$ & $57.59 \pm 7.95$ & $42.98 \pm 7.54$ & $75.30 \pm 14.8$ \\
+ & $20 \mu \mathrm{M} \mathrm{Pi}$ & 100 & $76.49 \pm 5.78$ & $76.60 \pm 5.82$ & $69.92 \pm 6.77$ & $87.34 \pm 2.42$ \\
\hline
\end{tabular}

${ }^{a}$ Estimation of mycorrhizal parameters was carried out according to Trouvelot and associates (1986). Mean values and standard deviations of five biological replicas of each treatment are shown. Myc = inoculation with Glomus intraradices, $\mathrm{Pi}=$ phosphate, $\mathrm{F}=$ frequency of colonization, $\mathrm{M}=$ colonization intensity of the whole root system, $\mathrm{SD}=$ standard deviation, $\mathrm{m}=$ colonization intensity within colonized root areas, $\mathrm{A}=$ arbuscule frequency within the whole roots system, and $\mathrm{a}=$ arbuscule frequency within arbuscule-containing root areas. 
increased cellular Pi due to symbiotic Pi transfer from fungus to the plant, since $M t P l \mathrm{D}$ as a PHO2-independent Pi stress marker shows decreased expression at low Pi in mycorrhizal roots as compared with nonmycorrhizal roots (discussed below). Pi concentration in shoots was higher in nonmycorrhizal plants than in mycorrhizal plants, probably an effect of increased shoot growth of mycorrhizal plants receiving the $20-\mu \mathrm{M}$ Pi treatment. Staining root samples of all four treatments revealed no mycorrhizal structures in the noninoculated samples, whereas inoculated roots displayed typical structures of a $G$. intraradices-M. truncatula AM symbiosis, namely intercellular hyphae, arbuscules, and vesicles. Comparison of mycorrhizal parameters between the inoculated roots of the two Pi treatments revealed that fertilization with $1 \mathrm{mM}$ Pi led to a $20 \%$ decrease in all parameters investigated, except for $\mathrm{F}$ (overall colonization frequency), which was $100 \%$ in both cases (Table 1). Hence, the high $\mathrm{Pi}$ conditions used in this experiment mainly suppressed arbuscule frequency and colonization intensity. In parallel, we quantified AM symbiosis by measuring three diagnostic markers for arbuscules and fungal colonization: i) MtPt4, a mycorrhizaspecific Pi transporter (Javot et al. 2007), ii) MtGst1, a glutathione- $S$ transferase-like mycorrhiza-specific gene (Wulf et al. 2003). and iii) ribosomal RNA of Glomus intraradices (Isayenkov et al. 2004). Using quantitative real-time polymerase chain reaction (qRT-PCR), transcripts of the two mycorrhizaspecific $M$. truncatula genes were not detected in samples of nonmycorrhizal roots. All three markers were highly expressed in mycorrhizal roots of both $\mathrm{Pi}$ treatments, with significantly lower expression in roots fertilized with $1 \mathrm{mM}$ Pi (Fig. 2). In accordance with data of the mycorrhiza estimation after root staining (described above), this indicates that the $1 \mathrm{mM}$ Pi treatment suppresses AM development.

\section{Characterization of the plant Pi status using diagnostic markers for Pi stress.}

As mentioned above, AM symbiosis led to increased Pi concentration in roots of Pi-depleted plants. To get further information about the Pi status of the plants used in this study, we


Fig. 2. Relative expression levels of mycorrhiza marker genes of Medicago truncatula (MtPt4, MtGst1) and Glomus intraradices rRNA, determined by quantitative reverse transcription-polymerase chain reaction (qRT-PCR). Phosphate (Pi)-depleted (-P) and Pi-repleted (+P) mycorrhizal (MYC) and nonmycorrhizal (NM) plants were harvested at 5 weeks postinoculation. Relative expression levels of target genes were determined by qRT-PCR using MtEf1 as reference gene. Values shown are mean \pm standard deviations of five biological replicates and three technical repetitions of each qRT-PCR reaction.
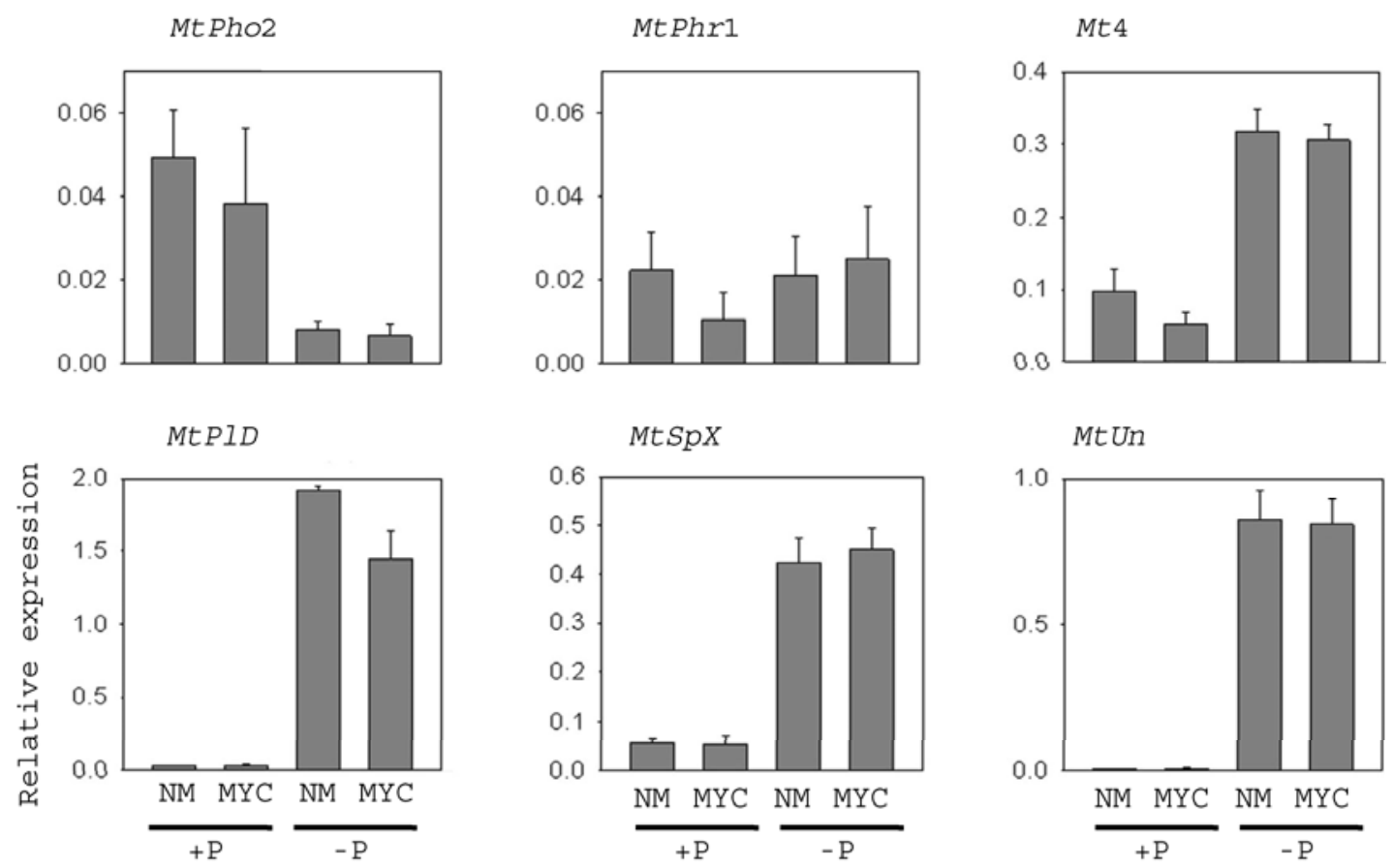

Fig. 3. Relative expression levels of phosphate (Pi) signaling and Pi-stress markers in mycorrhizal and nonmycorrhizal roots of Pi-repleted and Pi-depleted plants. Pi-depleted (-P) and -repleted ( $+\mathrm{P}$ ) mycorrhizal (MYC) and nonmycorrhizal (NM) plants were harvested at 5 weeks postinoculation. Relative expression levels of target genes were determined by quantitative reverse transcription-polymerase chain reaction (qRT-PCR) using $M t E f 1$ as reference gene. Values shown are mean \pm standard deviations of five biological replicates and three technical repetitions of each qRT-PCR reaction. 
quantified transcript levels of marker genes for Pi stress and homeostasis by qRT-PCR using roots of mycorrhizal and nonmycorrhizal plants of both Pi treatments. The markers include MtPho2, MtPhrl, and Mt4 involved in Pi homeostasis, as well as three Pi-stress markers, i.e., MtPlD, MtSpx, and MtUn (Hohnjec et al. 2005). The orthologs of MtSpx and MtUn have been shown to be PHO2-dependent in Arabidopsis, whereas the potential MtPlD ortholog in Arabidopsis is PHO2-independent (Bari et al. 2006). In agreement with previous reports, $M t P h r 1$ transcripts were not significantly dependent on Pi (Muller et al. 2007), whereas MtPho2 transcript levels decreased in Pidepleted plants (Fig. 3). Remarkably, MtPho2 transcript levels did not differ significantly between mycorrhizal and nonmycorrhizal plants. The three Pi-stress markers MtPlD, MtSpx, and $M t U n$ are strongly induced under Pi starvation. Notably, $M t 4$ transcripts were detected also under 1-mM Pi conditions, which is in contrast to previous reports that $M t 4$ was not detectable by Northern blot under Pi-sufficient conditions (Burleigh and Harrison 1997), indicating that the here-applied 1-mM Pi treatment could still represent mild Pi starvation for plants. The locally enhanced $\mathrm{Pi}$ in mycorrhizal roots of $\mathrm{Pi}$ depleted plants led to some repression of $M t P l D$ as compared with nonmycorrhizal roots, which is most probably a response to the locally increased $\mathrm{Pi}$ in mycorrhizal roots. The transcript levels of two other MtPHO2-dependent Pi-stress markers, MtSpx and MtUn, were not changed in response to mycorrhizal symbiosis in Pi-depleted plants, reflecting the unaltered MtPho2 levels. In summary, although AM symbiosis led to elevated Pi in roots (Fig. 1), this did not affect MtPho2 transcript levels. In conformity with this finding, expression of MtPHO2-dependent Pi-stress markers was also unaltered in mycorrhizal roots.

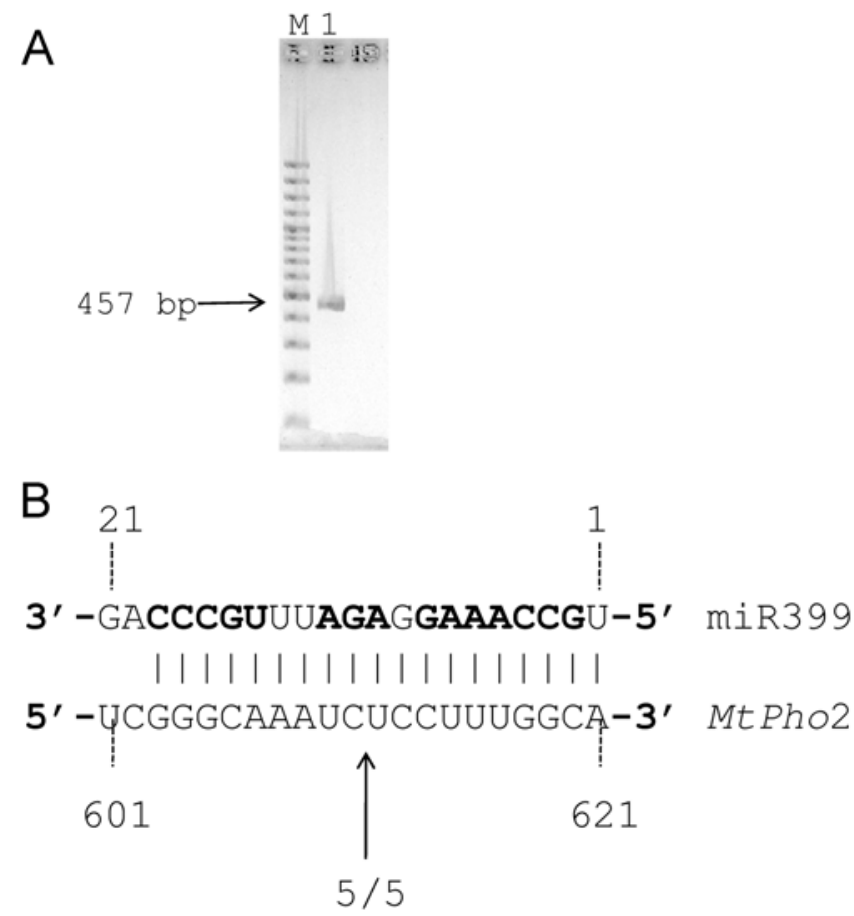

Fig. 4. Validation of miR399-mediated MtPho2 transcript cleavage. A modified RNA-ligase mediated-rapid amplification of cDNA ends (RLMRACE) using cDNA of mycorrhizal roots revealed one single polymerase chain reaction (PCR) product $\mathbf{A}, \mathbf{M}=$ DNA size marker, lane $1=$ amplification product after RLM-RACE. B, Sequencing of five cloned PCR products confirmed a predicted miR399 target site and transcript cleavage at position 611/612 of the MtPho2 cDNAin each of the five PCR products. Bold letters indicate conserved nucleotides within the Medicago truncatula mature miR399 sequences.
Identification of novel MIR399 genes in M. truncatula.

For a comprehensive analysis of the miR399 family with regard to AM symbiosis, we aimed for a more thorough identification and characterization of MIR399 genes in the model legume Medicago truncatula. We developed a miRNA prediction pipeline based on homology searches in the M. truncatula genome coupled to folding analysis of predicted RNA molecules. Based on the predicted free energy on secondary structures for known plant pre-miRNAs from miRBase, a cut-off of $-28 \mathrm{kcal} / \mathrm{mol}$ was introduced to distinguish true miRNAs from false positives. Sequences passing this requirement were trimmed back to the base of the outmost predicted stem. Trimmed sequences had to be at least 60 nucleotides in length, with a predicted structure containing a stem of at least 20 basepairs overlapping the region of similarity to the mature query sequence. Predicted miRNA precursor structures that contained more than three stem-loop branches were discarded. Applying this pipeline to mature plant miR399 sequences from miRBase Registry (release 13) resulted in the identification of 10 additional putative MIR399 genes in the M. truncatula genome. As expected, these MIR399 genes reside in so-far anonymous intergenic regions. Candidate miR399 sequences resulting from the prediction were manually compared with miR399 sequences deposited at the novel miRBase release 14 and were annotated. All of our predicted miR399 sequences appeared to be present in miRBase release 14 but without experimental confirmation. In order to verify our 10 in silico-predicted as well as the five previously (miRBase release 13) annotated miR399 transcripts, we designed specific primers for each of these 15 pri-MiR399. qRT-PCR analysis using cDNA of roots of Pi-depleted plants revealed specific amplification products for each of the primer pairs tested (data not shown). Supplementary Table S1 shows an overview of all our 15 experimentally confirmed MIR399 genes from M. truncatula.

\section{Confirmation of MtPho2 as a miR399 target in M. truncatula.}

In order to confirm that MtPho2 represents a target for miR399 in M. truncatula as it is described for A. thaliana, we carried out a modified rapid amplification of cDNA ends (RACE) experiment. The $5^{\prime}$ end of the MtPho2 cDNA was amplified from mycorrhizal roots grown under Pi-depleted conditions using a gene-specific primer, and one single band was obtained (Fig. 4). Cloning and sequencing of the PCR product revealed a miR399 binding and transcript cleavage site at position $611 / 612$ of the MtPho2 cDNA sequence.

In order to investigate if miR399 might have targets other than Pho 2 in $M$. truncatula, we used two target-prediction tools, the UEA Plant Small RNA Toolkit (Moxon et al. 2008) and the MiRU: Plant microRNA Potential Target Finder (Zhang 2005) (Supplementary Table S2). Both prediction tools confirmed $\mathrm{MtPho} 2$ as putative target after searching the DFCI (Dana Farber Cancer Institute) Medicago truncatula Gene Index 9.0 for miR399 targets. A second sequence (BG444416), a singleton with similarities to the MtPho2 5' untranslated region, was predicted to be a miR399 target from both prediction tools. A total of 10 other putative target sequences were predicted but none of them by both prediction tools.

\section{Mature miR399 accumulate to higher levels in mycorrhizal roots as compared with nonmycorrhizal roots under low Pi fertilization.}

We next wanted to determine whether pri-miR399 and mature miR399 levels are correlated with Pi and AM symbiosis status in M. truncatula. Pri-MiR399 were quantified in leaves and roots of mycorrhizal and nonmycorrhizal plants of both 1$\mathrm{mM}$ and 20- $\mu \mathrm{M}$ Pi fertilization treatments (Fig. 5). Most pri- 
MiR399 were only very weakly detectable in Pi-repleted plants. Highest pri-miR levels were measured in leaves of $\mathrm{Pi}$ depleted plants. Interestingly, five pri-MiR399 (miR399b, c, d, $\mathrm{m}$, and $\mathrm{o}$ ) were significantly higher in leaves of mycorrhizal Pi-depleted plants as compared with nonmycorrhizal plants. In roots, pri-miRNA399 levels were generally lower than in leaves, supporting the belief that leaves are the major site for miR399 transcription (Bari et al.; 2006; Pant et al.; 2008). Un- der low-Pi conditions, AM symbiosis in most cases led to decreased miR399 transcript levels mirroring the improved local Pi availability.

To investigate the abundance of mature miR399 molecules, we applied a stem-loop primer-based qRT-PCR. Sequence variation within the 15 mature miR399 sequences (Fig. 6) allowed us to design different stem-loop RT primers in order to distinguish four groups of mature MiR399 by PCR (miR399_GC,

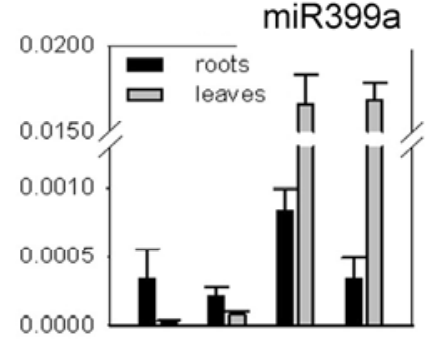

miR399d
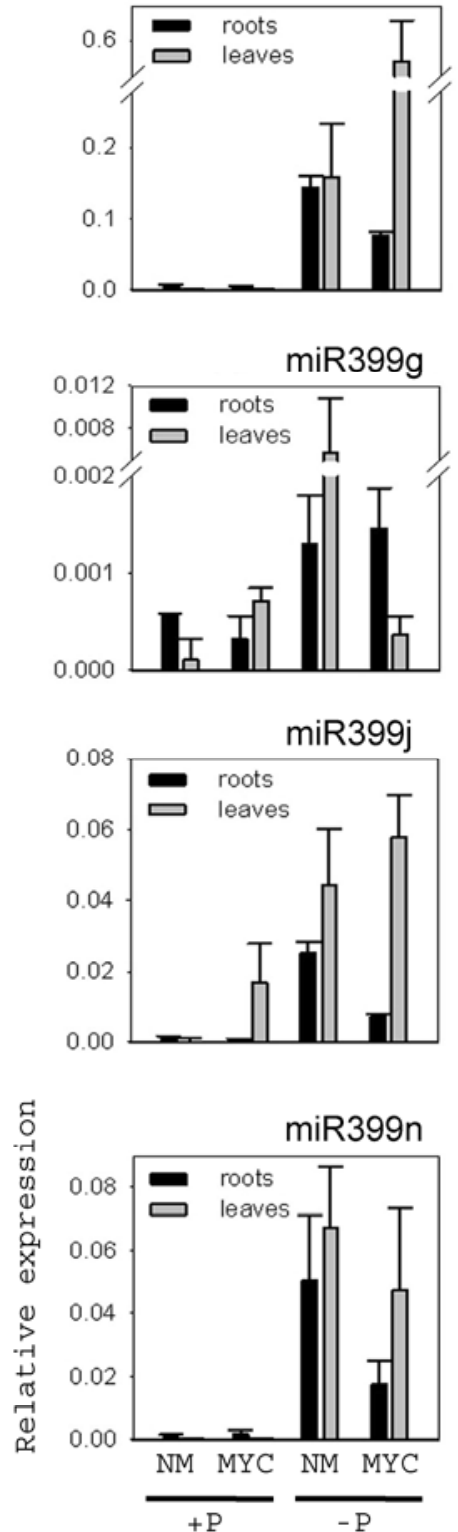

miR399b
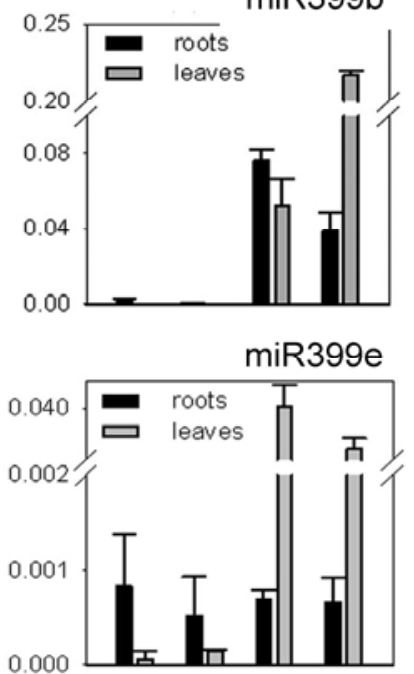

miR399h
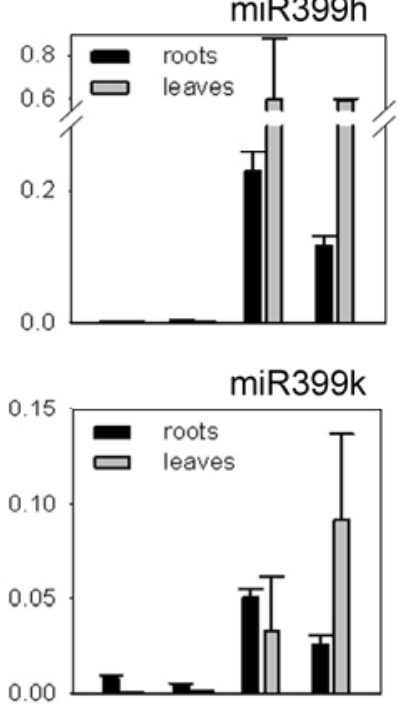

$\operatorname{miR} 3990$



miR399c
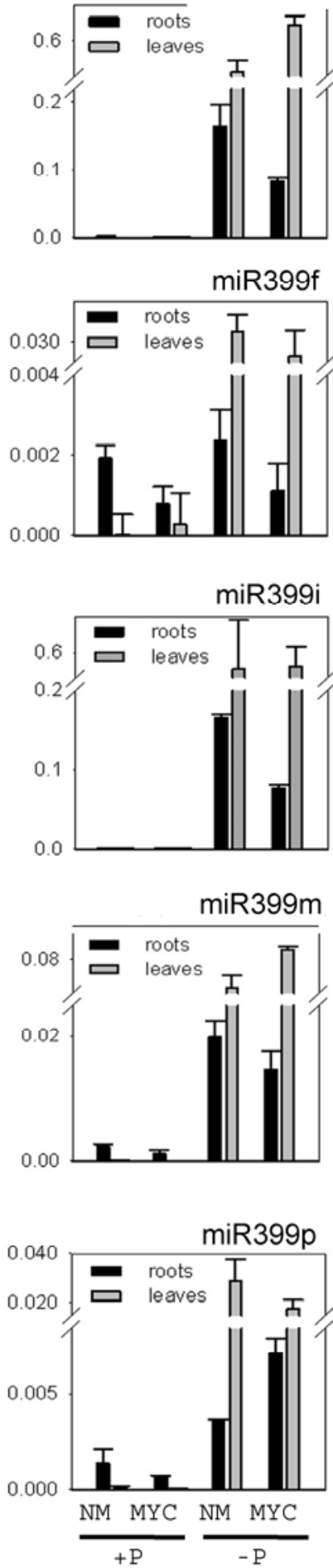

Fig. 5. Relative expression levels of 15 miR399 primary transcripts in roots and leaves of mycorrhizal and nonmycorrhizal phosphate (Pi)-depleted or Pirepleted plants. Pi-depleted (-P) and -repleted (+P) mycorrhizal (MYC) and nonmycorrhizal (NM) plants were harvested at 5 weeks postinoculation. Relative levels of miR399 primary transcripts were determined by quantitative reverse transcription-polymerase chain reaction using $M t E f 1$ as reference gene. Values are mean \pm standard deviations of five biological replicates and three technical repetitions. 
miR399_TT, miR399_TT/j, and miR399_GT/p). RNA of each tissue was reverse-transcribed using the appropriate RT primer. In parallel, identical amounts of RNA were used for a standard RT reaction, to allow normalization of the mature miR399 levels against reference transcripts. The expression levels of mature miR399 subfamilies were calculated as relative expression levels compared with MtEf1 as reference (Fig. 7). In Pi-repleted plants, mature MiR399 were very low and did not differ significantly between shoots and roots. In contrast, all four types of mature MiR399 could be detected at high levels in leaves and roots of Pi-depleted plants. Surprisingly, mycorrhizal roots displayed higher mature miR399 levels of all four subfamilies measured. We measured a statistically significant (two-way



analysis of variance, $P=0.001$ ) increased miR399j level in mycorrhizal roots as compared with nonmycorrhizal roots; for the three other miR399 subfamilies, no significant changes were observed in mycorrhizal roots. Hence, although AM symbiosis leads to locally improved Pi availability, the accumulation of mature miR399 is not decreased. In contrast, we observed a significant increase in mature miR399j. This and the expression pattern of MtPho2 and the Pi-stress markers led us to assume that miR399 might be involved in suppressing a $\mathrm{PHO} 2$ increase in mycorrhizal roots.
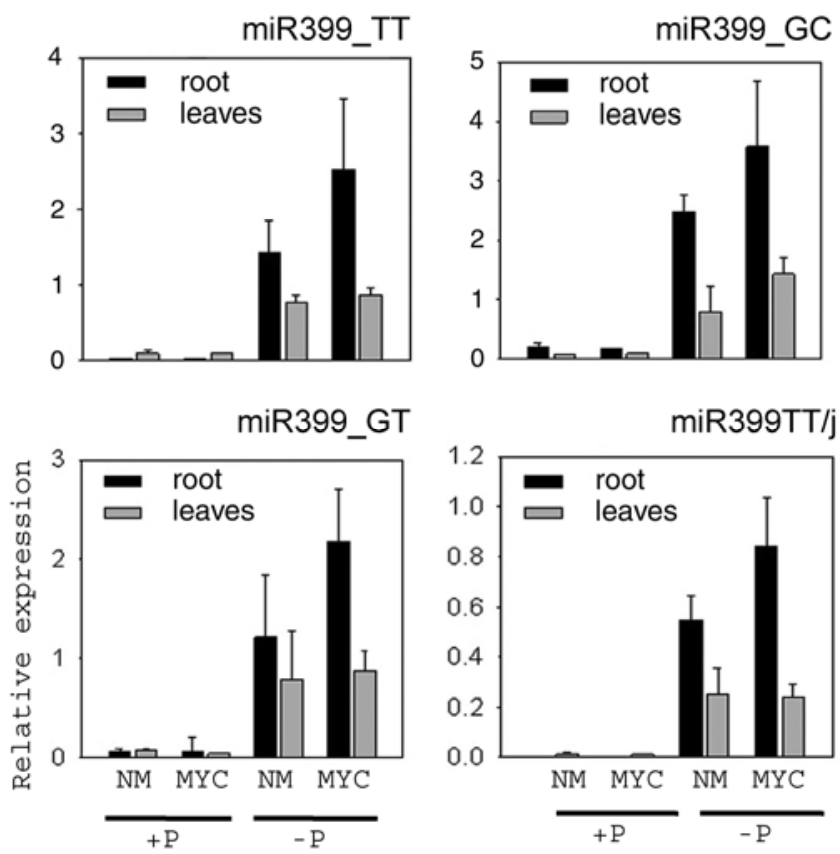

Fig. 7. Relative expression levels of four classes of mature miR399 in roots and leaves. Phosphate $(\mathrm{Pi})$-depleted $(-\mathrm{P})$ and -repleted $(+\mathrm{P})$ mycorrhizal (MYC) and nonmycorrhizal (NM) plants were harvested at 5 weeks postinoculation. Levels of mature miR399 of the subfamilies miR399_TT, miR399_GC, miR399_GT, and miR399_j were determined, and relative miR399 abundance was measured by quantitative reverse transcription (RT)-polymerase chain reaction after cDNA synthesis using specific stemloop (RT) primers for each miR399 subfamily. The values shown are mean \pm standard deviation of five biological replicates and three technical repetitions.
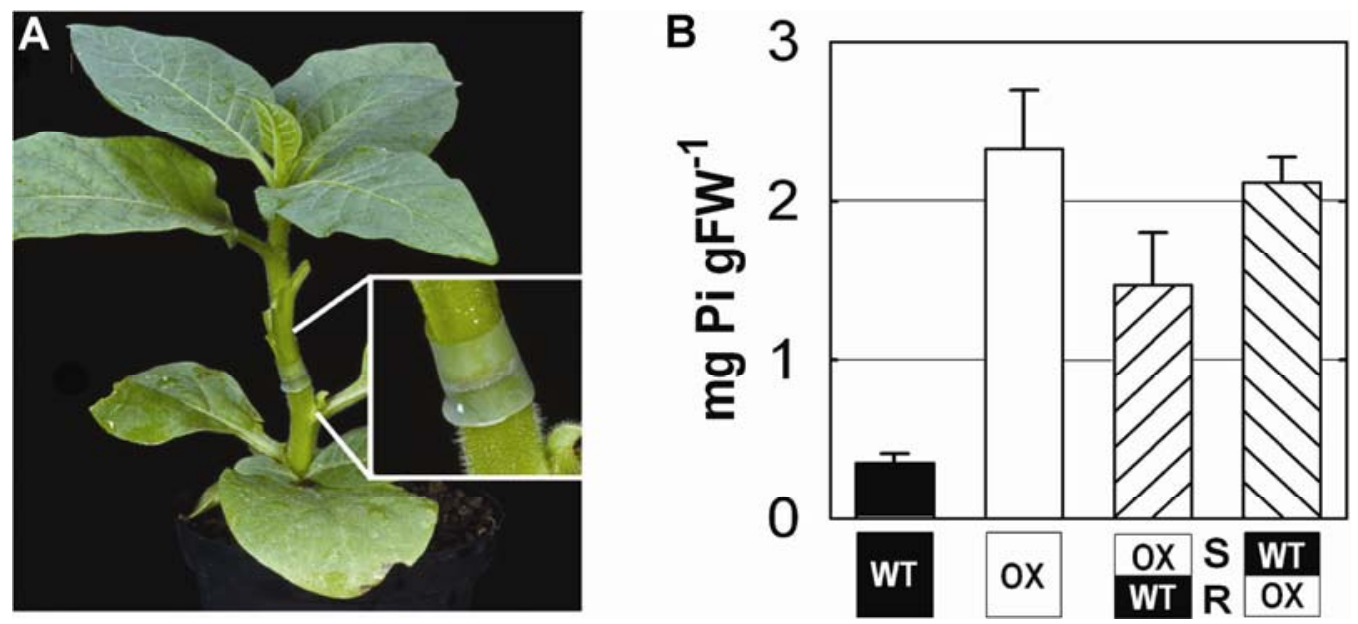

Fig. 8. Reciprocal grafting of miR399-overexpressing (OX) and wild-type (WT) tobacco plants. A, Aspect of a 62-day-old grafted tobacco plant. B, Phosphate (Pi) contents in leaves of 8-week-old, hydroponically grown WT (black bars), OX (white bars), and reciprocally grafted chimeras (hatched bars). Plants were fertilized with $1.5 \mathrm{mM}$ Pi. Root $(\mathrm{R})$ and shoot $(\mathrm{S})$ genotypes of the chimeric plants are depicted below the graph. Data are mean values \pm standard deviation of five replicates. 
Increased miR399 levels in mycorrhizal tobacco roots.

To test the hypothesis that MiR399 are regulators of Pi homeostasis during AM symbiosis and to investigate the influence of a constant Pi-starvation signal on AM symbiosis, we transformed tobacco plants with a construct leading to overexpression of miR399 (ath-miR399d) (Bari et al. 2006). Here, we used tobacco as an AM-capable plant model that can be easily transformed. In Arabidopsis, everexpression of miR399d leads to $\mathrm{Pi}$ accumulation in shoots under P-replete conditions and, thus, a pho2 phenocopy (Bari et al. 2006; Chiou et al. 2006). To see whether the ath-miR399d is biologically active in tobacco, we performed reciprocal grafting experiments with wild type (WT) and miR399-overexpressing (OX) tobacco plants (Fig. 8). High levels of Pi were found in shoots of miR399-OX plants. Moreover, grafting a WT shoot on OX roots or grafting a miR399-OX shoot on WT roots also led to $\mathrm{Pi}$ overaccumulation in shoots, demonstrating that i) miR399 overexpression in roots is sufficient to induce $\mathrm{Pi}$ accumulation in shoots and ii) that miR399 also is a long-distance signal in tobacco. Thus the observed phenotype is identical to the observed pho 2 or miR399-OX phenotype in Arabidopsis (Bari et al. 2006; Lin et al. 2008; Pant et al. 2008), showing that athmiR399d is biologically active in tobacco. Thus, miR399-OX tobacco plants display a constant high level of miR399 as systemic Pi-starvation signal and represent a suitable tool to analyze this miR399-born Pi-starvation signal concerning its involvement in the correlation between $\mathrm{Pi}$ status and AMsymbiosis development.

To analyze the effect of miR399 overexpression on AM symbiosis, plants were inoculated with G. intraradices, and roots were harvested 6 weeks after inoculation. To confirm the miR399 OX, we measured the mature miR399d expression by stem-loop PCR. Overexpression was confirmed for all transgenic plants (Supplementary Fig. S2). Apparently, the applied PCR conditions and primers were also able to detect endogenous tobacco mature miR399 expression. We observed a much stronger accumulation of mature miR399 in mycorrhizal Pidepleted WT roots as compared with nonmycorrhizal roots (Fig. 9), although soluble Pi contents of the leaves did not differ significantly (data not shown). This finding is consistent with our results in M. truncatula, in which strongest accumulation of mature miR399j was also detected in mycorrhizal roots under Pi-deplete conditions.

\section{MiR399 overexpression does not influence mycorrhizal colonization in tobacco.}

We next investigated whether miR399 overexpression leads to a phenotype with regard to root colonization by $G$. intraradices. A recent characterization of tobacco $\mathrm{Pi}$-transporter genes revealed the identification of $\mathrm{NtPt} 3(\mathrm{Pht} 1 ; 3)$ as $\mathrm{AM}$ upregulated as well as two transporters, NtPt4 and NtPt5 (Pht1;4 and Pht1;5, respectively), as AM-specifically expressed (Chen et al. 2007). We used the expression of these AM-regulated transporters as markers for an established AM symbiosis in the tobacco roots (Fig. 10). In contrast to the non-AM regulated transporter $\mathrm{NtPt} 1$, all three mycorrhiza-regulated $\mathrm{Pi}$ transporters were strongly increased in mycorrhizal roots of Pi-depleted plants. Expression levels in mycorrhizal roots of Pi-repleted plants were lower, mirroring the lower fungal colonization rate. This molecular phenotype did not differ between WT and miR399-OX plants. We next investigated the visual phenotype of miR399-OX plants with regard to root colonization by $G$. intraradices. To exclude local effects of Pi fertilization on the AM fungus, we used split-root systems. This experimental setup allowed the analysis of the systemic effect of $\mathrm{Pi}$ fertilization in one compartment on mycorrhizal colonization in the second compartment. Three weeks after inoculation with
G. intraradices, plants were harvested and roots of the inoculated compartment were stained for mycorrhizal structures. Evaluation of colonization intensity following methods described by Trouvelot and associates (1986) revealed a clear repression of colonization intensity in WT and in miR399-OX plants in Pi-repleted plants. Fertilization with $1 \mathrm{mM} \mathrm{Pi}$ decreased overall colonization intensity $(\mathrm{F})$ by $50 \%$ as compared with plants fertilized with $20 \mu \mathrm{M}$ Pi (data not shown). Arbuscule frequency was decreased more than 100-fold in Pi-repleted plants in both miR399-OX and WT plants. Hence, we could not observe an effect of miR399 per se with respect to establishment of AM-symbiosis in tobacco.

\section{DISCUSSION}

The aim of this work was to determine the correlation between miR399 expression, Pi homeostasis, and AM development in plants. A negative correlation between Pi status and development of AM symbiosis has been described in different plant-AM fungus combinations (Menge et al. 1978; Nagy et al. 2008; Sanders 1975). This is consistent with this study using $M$. truncatula or tobacco plants cultivated in split-root systems. In the latter case, fertilization with high Pi in one root compartment was able to suppress the AM symbiosis in the second root compartment. This indicates that either a systemic Pi availability signal suppresses AM symbioses or that a systemic Pi-starvation signal promoting symbiosis development is lacking. Evidence for a high Pi signal that is able to suppress AM symbiosis is provided by the fact that Pi-repleted roots become insensitive to the mycorrhizal signal LPC and the induction of mycorrhiza-inducible Pi transporters is repressed (Nagy et al. 2008). However, the Pi signal leading to suppression of the LPC signal is unknown.

MiR399 has been shown to act as a systemic Pi-starvation signal (Lin et al. 2008; Pant et al. 2008). Hence, one might speculate that MiR399 represent a systemic low-Pi signal pro-

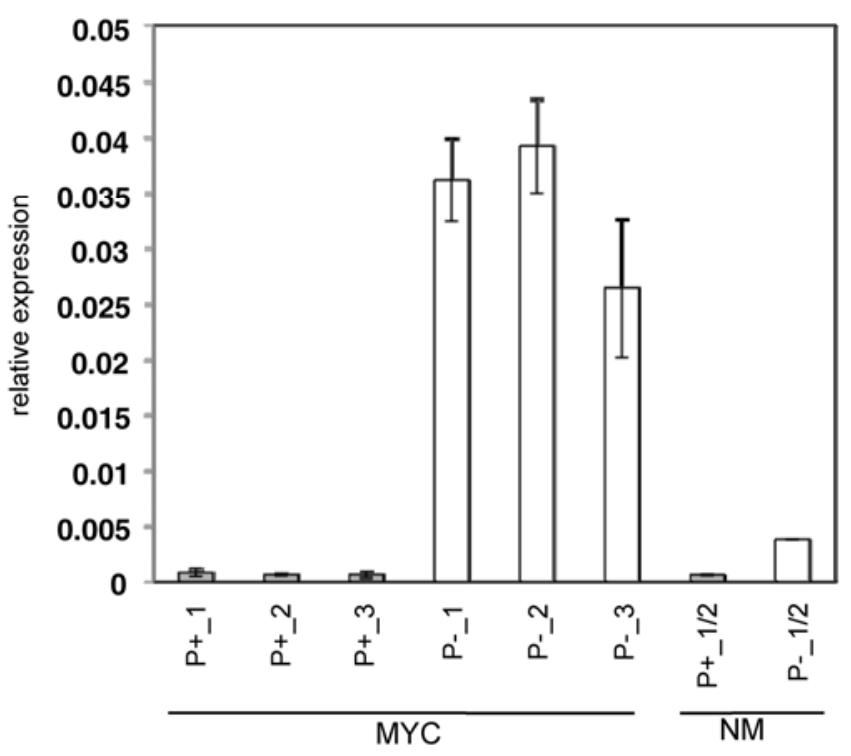

Fig. 9. Relative abundance of mature miR399 in roots of wild-type tobacco plants. Phosphate $(\mathrm{Pi})$-depleted $(\mathrm{P}-)$ and -repleted $(\mathrm{P}+)$ mycorrhizal (MYC) and nonmycorrhizal (NM) wild-type tobacco plants were harvested at 6 weeks postinoculation. Abundance of mature miR399d was measured by quantitative reverse transcription-polymerase chain reaction after cDNA synthesis using a miR399d-specific stem-loop (RT) primer. Three individual mycorrhizal plant were used for both Pi regimes (1 through 3). Two nonmycorrhizal plants of $\mathrm{P}+$ and $\mathrm{P}$ - were pooled (1/2). The values shown are mean \pm standard deviation from two technical repetitions. White bars represent expression under Pi-depleted conditions. 
moting or required for AM formation. On the other hand, they also could act as counter players of systemic Pi availability signals that suppress AM symbiosis. To test each of these assumptions, we analyzed the miR399 family in the AM-capable plant model Medicago truncatula and could experimentally confirm 10 novel MIR399 genes in this species. Recently, a bioinformatic approach for miR prediction from expressed sequence tags and genome survey sequences suggested five novel mem-
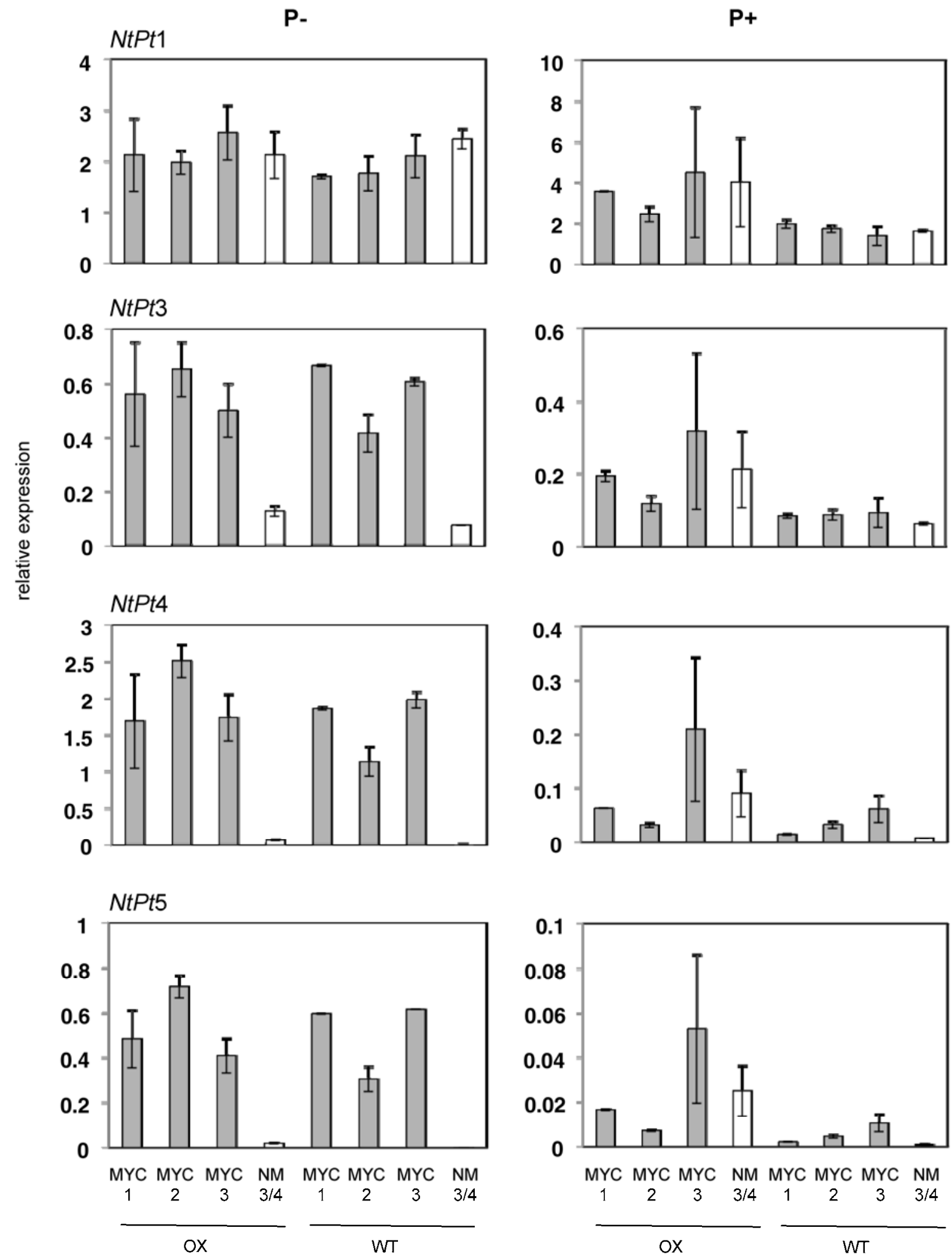

Fig. 10. Relative expression levels of tobacco phosphate $(\mathrm{Pi})$ transporter genes. Pi-depleted ( $\mathrm{P}-$; left panel) and -repleted (P+; right panel) mycorrhizal (MYC; gray bars) and nonmycorrhizal (NM; white bars) plants were harvested at 6 weeks postinoculation. The expression of NtPt2 as non-arbuscular mycorrhizal (AM) regulated Pi transporter as well as the expression of NtPt3, NtPt4, and NtPt5 as AM-regulated Pi transporters was measured. Individual plants were labeled by numbers. Two nonmycorrhizal plants of $\mathrm{P}+$ and $\mathrm{P}-$ were pooled (NM 3/4). Relative expression levels of target genes were determined in roots by quantitative reverse transcription-polymerase chain reaction using actin as reference gene. Values shown are mean \pm standard deviations of two technical repetitions. 
bers of the miR399 family in M. truncatula (Zhou et al. 2008). Of this data set, only one predicted miR399 (named 399i by Zhou and associates [2008]) is overlapping with our experimentally confirmed miR399 genes (miR399 miRBASE 14). In addition, a deep-sequencing approach of small RNAs from $M$. truncatula root apexes and nodules revealed 13 MIR399 genome loci, of which six precursor sequences are identical to our dataset (Lelandais-Briere et al. 2009). Hence, it is very likely that the miR399 family has even more members in $M$. truncatula. With 15 so-far confirmed members, the miR399 family currently represents the largest miRNA family in $M$. truncatula. This compares well to the number in rice and poplar, in which 11 and 12 members have been identified, respectively (Lindow et al. 2007). In A. thaliana, the miR399 family has only six members. Possibly, the low number of MiR399 in A. thaliana is related to the fact that A. thaliana, in contrast to $M$. truncatula, rice, and poplar, is not capable of enhancing its Pi uptake through symbiosis with AM fungi.

All 15 pri-miR399 members were strongly accumulating in Pi-depleted plants with higher transcript levels in leaves as compared with roots. This implies that leaves are the major sites of miR399 transcription. This was also observed in $A$. thaliana (Bari et al. 2006; Pant et al. 2008), in which the transcriptional induction of miR399 in leaves seems to be in response to a root-born systemic effect that has yet to be identified (Lin et al. 2008). In roots, we observed a decrease of primiR399 in mycorrhizal plants, implying that this is an effect of the AM-mediated improved local Pi availability. This is also in accordance with Lin and associates (2008), who postulated a local root-born signal regulating pri-MiR399 in roots. Surprisingly, we found five pri-MiR399 further induced in leaves of Pi-depleted mycorrhizal plants. On the other hand, in the $M$. truncatula-G. intraradices system under Pi-deplete conditions, we observed slightly decreased soluble Pi contents in shoots of mycorrhizal plants, which is most likely an effect of increased shoot fresh weights (data not shown). The phenomenon that AM symbiosis can dominate a plant's Pi supply without increasing total soluble Pi concentration in shoots has been described earlier (Smith et al. 2003). Moreover, the soluble Pi content is just a rough indicator of the actual Pi status; a precise determination of the Pi status requires ashing of the material or FTICR (Fourier transform ion cyclotron resonance mass spectrometry) to also determine chemically bound and insoluble Pi. Hence, whether the small change of soluble shoot Pi in the observed range and concentration has a direct impact in miR399 expression remains to be evaluated. In addition, as mentioned earlier, transcription levels of MiR399 in leaves are at least partially dependent on root-born signals, and the $\mathrm{Pi}$ contents in mycorrhizal roots were higher than in nonmycorrhizal roots. Hence, although we currently cannot exclude the possibility that the observed decrease in soluble Pi content in leaves of mycorrhizal plants causes an induction of miR399 transcription, it seems to be unlikely.

Mature miR399 accumulation was higher in roots than in shoots, consistent with their role as signaling molecules transported from shoots to roots, in which they negatively regulate PHO2 (Lin et al. 2008; Pant et al. 2008). We could experimentally confirm a miR399-mediated cleavage of the MtPho2 tran-

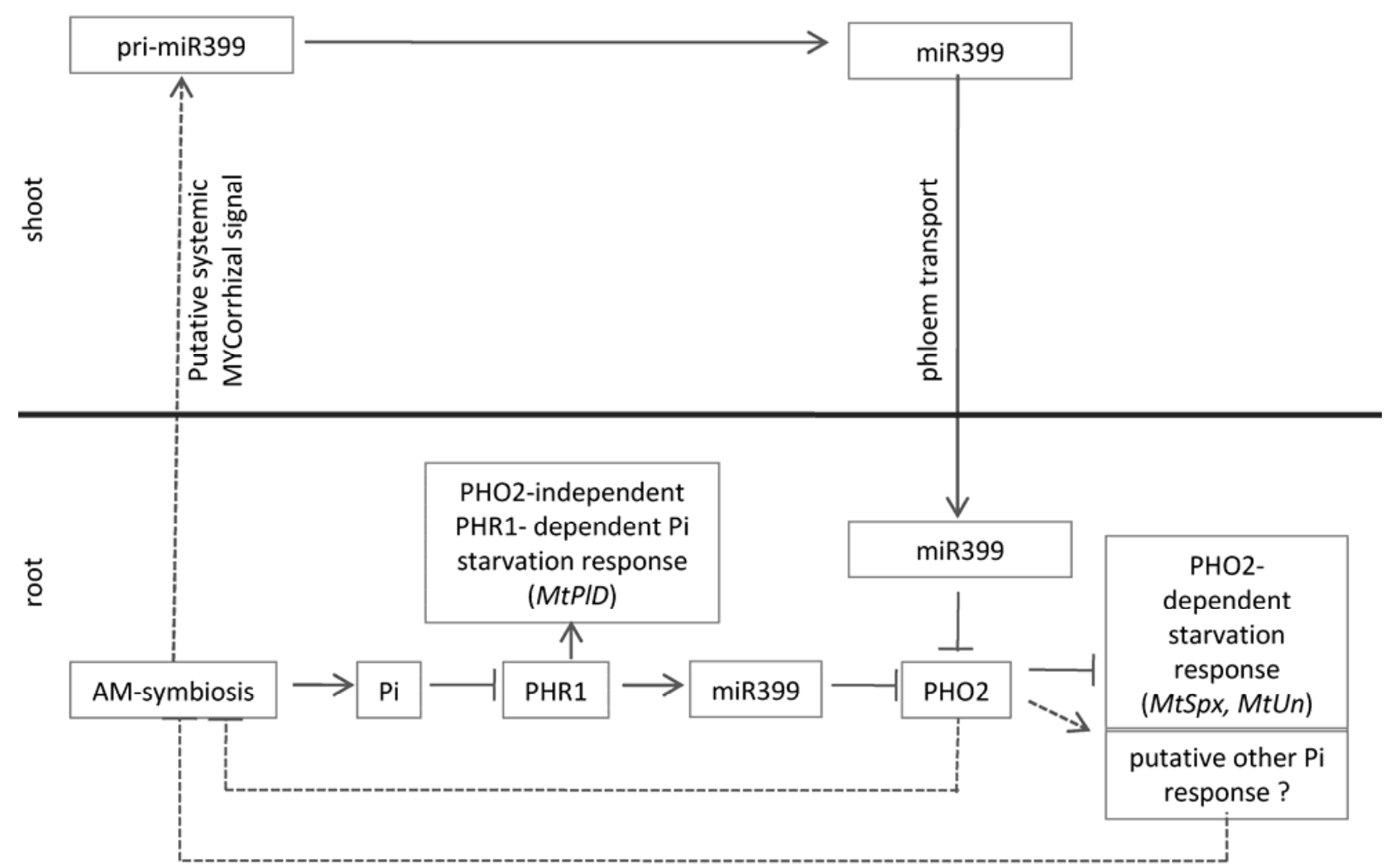

Fig. 11. A hypothetical model for the role of miR399 during arbuscular mycorrhizal (AM) symbiosis. AM symbiosis leads to a local phosphate (Pi) increase in colonized roots. Hence, PHR1 will be downregulated and, consistently, PHR1-depending Pi-stress markers and pri-miR399 synthesis are repressed. In parallel, a hypothetical mycorrhizal signal leads to increased pri-miR399 synthesis in shoots, which leads to increased accumulation of mature miR399 in mycorrhizal roots after phloem transport. Here, miR399 suppress PHO2. This leads to unaltered levels of Pho2 and PHO2-dependent Pi-stress markers in mycorrhizal roots, although Pi is locally increased and miR399 transcript levels are decreased. This suggests that, in mycorrhizal roots, increased miR399 levels keep MtPho2 expression and activity low, which otherwise would increase in response to symbiotic Pi uptake. One possible reason for this phenomenon is that PHO2 or other PHO2-dependent high-Pi responses might be involved in the suppression of AM symbiosis in Pi-repleted plants. Thus, an induction of PHO2 due to a symbiotically provided Pi increase in colonized roots has to be prevented by miR399 action in order to sustain mycorrhizal symbiosis. Yet unknown pathways are indicated as dashed lines. 
script at position 611/612 in mycorrhizal M. truncatula roots, indicating that the miR399-PHO2 regulation pathway is conserved in $M$. truncatula and active in mycorrhizal roots. Recently, four miR399-cleavage sites in MtPho2 transcript were found in RNA of whole Pi-stressed seedlings (Jagadeeswaran et al. 2009). Hence, it might be anticipated that miR399-mediated MtPho 2 cleavage patterns are tissue specific.

Surprisingly, we found an increase of mature miR399 in mycorrhizal roots as compared with nonmycorrhizal roots in M. truncatula and tobacco. In addition, five pri-MiR399 showed increased transcription levels in leaves of mycorrhizal plants. Hence, the observed expression pattern of pri- and mature miR399 suggests an AM symbiosis-related signal that appears to increase miR399 transcription in leaves and consequentially increased abundance of mature miR399 in mycorrhizal roots. The increased miR399 abundance in mycorrhizal roots is mirrored by the expression pattern of Pi-stress marker genes; MtPho2 transcripts as miR399 targets remained low although the Pi content is increased in mycorrhizal roots and, consistently, PHO2-dependent Pi-stress markers do not also change mycorrhizal roots. In contrast, transcript levels of $M t P l \mathrm{D}$, which is PHO2-independent, decreased in mycorrhizal roots. This suggests that, in mycorrhizal roots, increased miR399 levels keep MtPho2 expression and activity low, which otherwise would increase in response to symbiotic $\mathrm{Pi}$ uptake. Figure 11 shows a hypothetical model for the regulation and role of miR399 during mycorrhizal symbiosis.

To further strengthen our hypothesis that miR399 members are suppressing high $\mathrm{Pi}$ signals in mycorrhizal roots rather than being a Pi-starvation signal capable, per se, of improving AM colonization, we characterized the mycorrhizal phenotype of miR399-OX tobacco plants. We decided to use tobacco as the AM-capable plant model, because it is more suitable for transformation approaches than M. truncatula. Micrografting experiments confirmed a strong $\mathrm{Pi}$ overaccumulation in leaves of ath-miR399d-OX tobacco plants, demonstrating that the athmiR399d is biologically active in tobacco and that these OX plants are a suitable model to study the influence of a constant Pi-starvation signal on the AM-symbiosis. Transcription levels of mycorrhiza-regulated Pi transporters (Chen et al. 2007) as well as estimation of mycorrhizal structures by light microscopy revealed the same repression of mycorrhizal symbiosis under Pi-replete conditions in miR399-OX and WT plants. This is consistent with our assumption that an internal miR399-based low Pi signal alone is not enough to enhance AM development in Pi-repleted plants. Moreover, constant high miR399 levels are not able to bypass AM suppression in Pi-repleted plants. Both conclusions are in accordance to the observation that a high Pi signal is epistatic to the mycorrhizal signal LPC (Nagy et al.). However, the corresponding high Pi signal remains to be identified and whether additional upstreamacting components of the $\mathrm{Pi}$-signaling pathway are involved in this phenomenon remains to be answered. Recent studies in Arabidopsis revealed at least four additional miR with strong Pi status-dependent expression (Pant et al. 2009). This finding indicates that the regulation of $\mathrm{Pi}$ stress and homeostasis is remarkably widespread. Moreover, one novel miRNA (miR2111) was also detected with Pi status-dependent abundance in rapeseed (Brassica napus) phloem sap, indicating that it might be a new potential long-distance signal that should be tested for its involvement in AM symbiosis establishment.

\section{MATERIALS AND METHODS}

\section{Prediction of miR399 genes of Medicago truncatula.}

Plant miRNAs exhibit great variation in precursor length, and frequently, only the mature sequence is conserved between related species (Rhoades et al. 2002). Therefore, mature miR399 sequences were used as queries to find corresponding potential mature miR399 sequences in the $M$. truncatula genome. The program SSEARCH (Pearson 2000) was used for this purpose, with an $e$-value cutoff of 0.5 . Genomic hits to mature miRNA399 were extended by 400 nucleotides in both directions. The folding potential of these sequences was assessed using RNAfold (Zuker et al. 1991).

\section{Plant material, inoculation, and growth conditions.}

Medicago truncatula cv. Jemalong line A17 seeds were incubated with sulfuric acid, followed by repeated washings with distilled water. Afterwards, surface sterilization was carried out using $6 \%$ (vol/vol) sodium hypochlorite for $8 \mathrm{~min}$. After rinsing with sterile water, seeds were germinated on water agar at $4^{\circ} \mathrm{C}$ for $48 \mathrm{~h}$, followed by a 3 -day incubation at room temperature. Seedlings were placed in sand and expanded clay (1:2). Inoculation with Glomus intraradices (BEG141) was carried out using 10\% (vol/vol) inoculum (Biorize, Dijon, France).

All plants were grown in growth rooms under a $16 \mathrm{~h}$ light (photosynthetically active radiation, $300 \mu \mathrm{mol}$ photons $\mathrm{m}^{-2} \mathrm{~s}^{-1}$ ), 8 -h dark $\left(22^{\circ} \mathrm{C}\right), 60 \%$ relative humidity regime. Plants were fertilized twice a week with $25 \mathrm{ml}$ of Hoagland's solution (Hoagland and Arnon 1950) containing either $20 \mu \mathrm{M}$ or $1 \mathrm{mM}$ $\mathrm{KH}_{2} \mathrm{PO}_{4}$. Plants were harvested 5 weeks after inoculation. For each experiment, five biological replicates containing two plants were harvested. Samples of the roots were stained with trypan blue in order to estimate colonization intensity according to Trouvelot and associates (1986).

\section{Soluble Pi measurement.}

Pi levels were measured using a colorimetric method (Ames 1966). Tissue was ground in liquid nitrogen to a fine powder, and $20 \mathrm{mg}$ of tissue powder were incubated in $200 \mu \mathrm{l}$ of 25 $\mathrm{mM} \mathrm{NaOH}$. Samples were incubated at $95^{\circ} \mathrm{C}$ for $30 \mathrm{~s}$, and 200 $\mu \mathrm{l}$ of $25 \mathrm{mM} \mathrm{HCl}$ and $100 \mu \mathrm{l}$ of Tween-20 were added. Samples were mixed and centrifuged for $4 \mathrm{~min}$ at 9,000 rpm. Supernatant ( 3 to $15 \mu \mathrm{l}$ ) was mixed with $15 \mu$ l of water, $100 \mu \mathrm{l}$ of $\mathrm{HCL}$, and $100 \mu \mathrm{l}$ of color reagent (1 volume of $4.2 \%$ $\left(\mathrm{NH}_{4}\right)_{6} \mathrm{Mo}_{7} \mathrm{O}_{24} \cdot \mathrm{H}_{2} \mathrm{O}$ in $5 \mathrm{~N} \mathrm{HCl}$, three volumes of malachite green dye in water). The reaction was incubated at room temperature for $15 \mathrm{~min}$ before $100 \mu \mathrm{l}$ of $1.5 \%$ Tween-20 were added. The absorbance at $660 \mathrm{~nm}$ was measured after a second incubation of $15 \mathrm{~min}$. The Pi concentration in the samples was determined against a calibration curve.

\section{Generation of ath-miR399d overexpressing tobacco plants.}

The construct for constitutively overexpressing the athmiR399d was created by cloning a 289-bp fragment containing ath-miR399d precursor sequence into CaMV:35S promoter containing vector pMDC32 (Bari et al. 2006; Pant et al. 2008). Agrobacterium tumefaciens GV3101 containing the construct was used for transformation of tobacco (Nicotiana tabacum cv. SNN) plants. In brief, the cut pieces of the tobacco leaves were washed in bacterial suspension containing the construct and placed on Murashige Skoog (MS) media with 2\% agar, followed by incubation for 2 days in the dark. The leaf pieces were then transferred into MS medium with $1.6 \%$ glucose + 6-benzylaminopurine $(1 \mathrm{mg} / \mathrm{ml})+$ naphthylacetic acid $(0.2 \mathrm{mg} / \mathrm{ml})+$ claforan $(300 \mathrm{mg} / \mathrm{liter})+$ hygromycin $(10 \mathrm{mg} / \mathrm{ml})$. The medium was changed every 7 to 10 days, and the regenerated calli were transferred into glass pots with the same medium. The shoots were cut and transferred onto MS media with $2 \%$ sucrose + claforan $(250 \mathrm{mg} / \mathrm{liter})$. The tobacco seedlings were transferred to the greenhouse and overexpression of athmiR399d was confirmed by qRT-PCR. 
Tobacco grafting.

MiR399 OX and WT tobacco plants were grown in hydroponic systems providing $1.5 \mathrm{mM}$ Pi (Scheible et al. 2004). At 40 days old, plants were reciprocally grafted using silicon tubing. Grafted plants were covered to maintain high humidity until the graft junction had healed. Successfully grafted plants were used for Pi-content measurements when plants were 8 weeks old.

\section{Mycorrhizal inoculation \\ of ath-miR399d overexpressing and WT tobacco.}

Seeds of wild-type and ath-miR399d-overexpressing plants were surface sterilized by incubation for $2 \mathrm{~min}$ in $70 \%$ ethanol, were washed with sterile water, and treated for 6 min with $10 \%$ Na-hypochlorite. After a second washing step, seeds were germinated on wet filter paper in petri dishes at room temperature in the dark. To select for overexpressing plants, $20 \mu \mathrm{g}$ of hygromycin per milliliter was added to the water. After 10 days, the seedlings were planted into quartz sand in a growth chamber and were fertilized every two days with Hoagland's solution containing either $20 \mu \mathrm{M}$ Pi (-Pi) or $2 \mathrm{mM}(+\mathrm{Pi})$. Half of the plants were incoculated with $10 \%$ (vol/vol) Glomus intraradices inoculum. Roots were harvested after 6 weeks und were used for qRT$\mathrm{PCR}$, in order to quantify the expression of mycorrhiza-regulated Pi-transporter genes. For light microscopy analysis, plants were transferred after 17 days to split root pots containing quartz sand and $10 \%$ (vol/vol) Glomus intraradices inoculum in one compartment. Roots in the nonmycorrhiza compartment were fertilized with Hoagland's solution containing either $20 \mu \mathrm{M}$ Pi $(-\mathrm{Pi})$ or $2 \mathrm{mM}(+\mathrm{Pi})$. Three weeks after inoculation, plants were harvested. For the estimation of mycorrhiza parameters according to Trouvelot and associates (1988), we used four biological replicate plants per treatment (WT, overexpression mutant, $-\mathrm{Pi},+\mathrm{Pi})$.

\section{RNA extraction and qRT-PCR.}

Roots and shoot tissue were harvested and frozen in liquid nitrogen. RNA was isolated using the mirVana miRNA kit (Ambion, Austin, TX, U.S.A.) and was quantified using a NanoDrop spectrophotometer (Thermo Scientific, Wilmington, DE, U.S.A.). The RNA integrity was proven using a Bioanalyzer (Agilent, Palo Alto, CA, U.S.A.), and all RNA samples displayed RIN (RNA integrity numbers) $>8$. RNA samples were DNase treated using the TURBO DNA-free kit (Ambion), and $1 \mu \mathrm{g}$ of RNA was reverse transcribed using superscript III reverse transcriptase (Invitrogen, Karlsruhe, Germany). qPCR measurements were performed using the ABI PRISM 7900 HT sequence detection system (Applied Biosystems, Foster City, CA, U.S.A.) using Power SYBR green (Applied Biosystems). cDNA samples were diluted 1:10, and $1 \mu \mathrm{l}$ of diluted cDNA was used as template in 10- $\mu$ PCR reactions containing $2.5 \mu \mathrm{M}$ primer. qRT-PCR was carried out in optical 384-well plates with the following PCR regime: $50^{\circ} \mathrm{C}$ for 2 $\min , 95^{\circ} \mathrm{C}$ for $10 \mathrm{~min}, 40$ cycles of $95^{\circ} \mathrm{C}$ for $15 \mathrm{~s}$ and $60^{\circ} \mathrm{C}$ for $1 \mathrm{~min}$. Melting curve analysis was carried out after each amplification to exclude unspecific amplifications from the analysis. Data analysis was performed using the SDS2.2.1 software (Applied Biosystems). The threshold was set at 0.2. As constitutively expressed $M$. truncatula reference gene, we used the transcription elongation factor EF1 $\alpha$ (Wulf et al. 2003; Kakar et al. 2008) for the normalization of expression levels of the genes of interest. The amplification efficiencies (E) were calculated using the LinRegPCR program (Ramakers et al. 2003). For normalization, the reference gene cycle threshold $\left(\mathrm{C}_{\mathrm{T}}\right)$ value was subtracted from the $\mathrm{C}_{\mathrm{T}}$ value of the gene of interest, yielding a $\Delta \mathrm{C}_{\mathrm{T}}$ value. The relative expression levels of the target genes were calculated using the formula $(1+\mathrm{E})^{\Delta \mathrm{CT}}$ (Pfaffl
2001). Three technical replicates and five independent biological replicates were measured in each experiment. For quantification of mature miR399, total RNA was reverse transcribed using stem-loop primers and MultiScribe reverse transcriptase (Applied Biosystems). In brief, $1 \mu \mathrm{g}$ of RNA was mixed with $1 \mu \mathrm{l}$ of $10 \mathrm{mM}$ dNTPs, $1 \mu \mathrm{l}$ of $2.5 \mu \mathrm{M}$ stemloop RT primer, and RNase-free water to a final volume of $36.5 \mu \mathrm{l}$, and the mixture was heated to $65^{\circ} \mathrm{C}$ for $5 \mathrm{~min}$ before being chilled on ice. Then, $10 \mu \mathrm{l}$ of $5 \times$ first-strand buffer, 2 $\mu \mathrm{l}$ of $0.1 \mathrm{M}$ dithiothreitol, $0.5 \mu \mathrm{l}$ of RNase inhibitor, and $1 \mu \mathrm{l}$ of MultiScribe reverse transcriptase were added. The reaction mixture was incubated for $30 \mathrm{~min}$ at $16^{\circ} \mathrm{C}$, followed by an elongation reaction at $42^{\circ} \mathrm{C}$ for $30 \mathrm{~min}$ and an incubation at $85^{\circ} \mathrm{C}$ for $5 \mathrm{~min}$. Mature miRNAs were quantified using qRTPCR as described above.

\section{miR399 target validation \\ by modified RNA ligase-mediated (RLM)-RACE.}

RNA of mycorrhizal Pi-depleted roots was reverse transcribed using gene-specific primers (Pho2_outer: 5' to 3', CGTATGTA TTGCAATCAACAAC; Pho2_inner: 5' to 3', GATGAGTCGA TACCAACTTC), using the FirstChoice RLM-RACE kit (Applied Biosystems) according to the manufacturer's instructions, except that the RNA was directly ligated to the 5' RACE adapter without the RNA processing steps (calf intestine phosphatase and tobacco acid pyrophosphatase treatment). The PCR products were cloned into the TOPO TA cloning kit (Invitrogen) and were sequenced.

\section{ACKNOWLEDGMENTS}

This work was supported by the Max Planck Society.

\section{LITERATURE CITED}

Allen, E., Xie, Z., Gustafson, A. M., and Carrington, J. C. 2005. microRNAdirected phasing during trans-acting siRNA biogenesis in plants. Cell 121:207-221.

Ames, B. N. 1966. Assay of inorganic phosphate, total phosphate and phosphatases. Methods Enzymol. 8:115-118.

Aung, K., Lin, S. I., Wu, C. C., Huang, Y. T., Su, C. L., and Chiou, T. J. 2006. pho2, a phosphate overaccumulator, is caused by a nonsense mutation in a microRNA399 target gene. Plant Physiol. 141:10001011.

Bari, R., Datt Pant, B., Stitt, M., and Scheible, W. R. 2006. PHO2, microRNA399, and PHR1 define a phosphate-signaling pathway in plants. Plant Physiol. 141:988-999.

Bucher, M. 2007. Functional biology of plant phosphate uptake at root and mycorrhiza interfaces. New Phytol. 173:11-26.

Burleigh, S. H., and Harrison, M. J. 1997. A novel gene whose expression in Medicago truncatula roots is suppressed in response to colonization by vesicular-arbuscular mycorrhizal (Vam) fungi and to phosphate nutrition. Plant Mol. Biol. 34:199-208.

Chen, A., Hu, J., Sun, S., and Xu, G. 2007. Conservation and divergence of both phosphate- and mycorrhiza-regulated physiological responses and expression patterns of phosphate transporters in solanaceous species. New Phytol. 173:817-831.

Chiou, T. J., Aung, K., Lin, S. I., Wu, C. C., Chiang, S. F., and Su, C. L. 2006. Regulation of phosphate homeostasis by MicroRNA in Arabidopsis. Plant Cell 18:412-421.

Drissner, D., Kunze, G., Callewaert, N., Gehrig, P., Tamasloukht, M., Boller, T., Felix, G., Amrhein, N., and Bucher, M. 2007. Lyso-phosphatidylcholine is a signal in the arbuscular mycorrhizal symbiosis. Science 318:265-268.

Franco-Zorrilla, J. M., Valli, A., Todesco, M., Mateos, I., Puga, M. I., Rubio-Somoza, I., Leyva, A., Weigel, D., Garcia, J. A., and Paz-Ares, J. 2007. Target mimicry provides a new mechanism for regulation of microRNA activity. Nat. Genet. 39:1033-1037.

Glassop, D., Smith, S. E., and Smith, F. W. 2005. Cereal phosphate transporters associated with the mycorrhizal pathway of phosphate uptake into roots. Planta 222:688-698.

Harrison, M. J. 1999. Molecular and cellular aspects of the arbuscular my- 
corrhizal symbiosis. Ann. Rev. Plant Physiol. Plant Mol. Biol. 50:361389

Harrison, M. J., Dewbre, G. R., and Liu, J. 2002. A phosphate transporter from Medicago truncatula involved in the acquisition of phosphate released by arbuscular mycorrhizal fungi. Plant Cell 14:2413-2429.

Hause, B., and Fester, T. 2005. Molecular and cell biology of arbuscular mycorrhizal symbiosis. Planta 221:184-196.

Hoagland, D. R., and Arnon, D. I. 1950. The water-culture method of growing plants without soil. California Agricultural Experiment Station Circular 347, Berkeley, CA, U.S.A.

Hohnjec, N., Vieweg, M. F., Puhler, A., Becker, A., and Kuster, H. 2005. Overlaps in the transcriptional profiles of Medicago truncatula roots inoculated with two different Glomus fungi provide insights into the genetic program activated during arbuscular mycorrhiza. Plant Physiol. 137:1283-1301.

Isayenkov, S., Fester, T., and Hause, B. 2004. Rapid determination of fungal colonization and arbuscule formation in roots of Medicago truncatula using real-time (RT) PCR. J. Plant Physiol. 161:1379-1383.

Jagadeeswaran, G., Zheng, Y., Li, Y. F., Shukla, L. I., Matts, J., Hoyt, P., Macmil, S. L., Wiley, G. B., Roe, B. A., Zhang, W., and Sunkar, R. 2009. Cloning and characterization of small RNAs from Medicago truncatula reveals four novel legume-specific microRNA families. New Phytol. 184:85-98.

Javot, H., Penmetsa, R. V., Terzaghi, N., Cook, D. R., and Harrison, M. J. 2007. A Medicago truncatula phosphate transporter indispensable for the arbuscular mycorrhizal symbiosis. Proc. Natl. Acad. Sci. U.S.A. 104:1720-1725.

Kakar, K., Wandrey, M., Czechowski, T., Gaertner, T., Scheible, W. R. Stitt, M., Torres-Jerez, I., Xiao, Y., Redman, J. C., Wu, H. C., Cheung, F., Town, C. D., and Udvardi, M. K. 2008. A community resource for high-throughput quantitative RT-PCR analysis of transcription factor gene expression in Medicago truncatula. Plant Methods 4:18.

Lelandais-Briere, C., Naya, L., Sallet, E., Calenge, F., Frugier, F., Hartmann, C., Gouzy, J., and Crespi, M. 2009. Genome-wide Medicago truncatula Small RNA analysis revealed novel MicroRNAs and isoforms differentially regulated in roots and nodules. Plant Cell 21:2780-2796

Lin, S. I., Chiang, S. F., Lin, W. Y., Chen, J. W., Tseng, C. Y., Wu, P. C., and Chiou, T. J. 2008. Regulatory network of microRNA399 and PHO2 by systemic signaling. Plant Physiol. 147:732-746.

Lindow, M., Jacobsen, A., Nygaard, S., Mang, Y., and Krogh, A. 2007. Intragenomic matching reveals a huge potential for miRNA-mediated regulation in plants. PLoS Comput. Biol. 3:e238.

Maeda, D., Ashida, K., Iguchi, K., Chechetka, S. A., Hijikata, A., Okusako, Y., Deguchi, Y., Izui, K., and Hata, S. 2006. Knockdown of an arbuscular mycorrhiza-inducible phosphate transporter gene of Lotus japonicus suppresses mutualistic symbiosis. Plant Cell Physiol. 47:807-817.

Menge, J. A., Steirle, D., Bagyaraj, D. J., Johnson, E. L. V., and Leonard, R. T. 1978. Phosphorous concentrations in plants responsible for the inhibition of mycorrhizal infection. New Phytol. 80:575-578.

Moxon, S., Jing, R., Szittya, G., Schwach, F., Rusholme Pilcher, R. L., Moulton, V., and Dalmay, T. 2008. Deep sequencing of tomato short RNAs identifies microRNAs targeting genes involved in fruit ripening. Genome Res. 18(10):1602-1609

Muller, R., Morant, M., Jarmer, H., Nilsson, L., and Nielsen, T. H. 2007. Genome-wide analysis of the Arabidopsis leaf transcriptome reveals interaction of phosphate and sugar metabolism. Plant Physiol 143:156-171.

Nagy, R., Karandashov, V., Chague, V., Kalinkevich, K., Tamasloukht, M. Xu, G., Jakobsen, I., Levy, A. A., Amrhein, N., and Bucher, M. 2005. The characterization of novel mycorrhiza-specific phosphate transporters from Lycopersicon esculentum and Solanum tuberosum uncovers functional redundancy in symbiotic phosphate transport in solanaceous species. Plant J. 42:236-250.

Nagy, R., Drissner, D., Amrhein, N., Jakobsen, I., and Bucher, M. 2008. Mycorrhizal phosphate uptake pathway in tomato is phosphorus-repressible and transcriptionally regulated. New Phytol. 181:1602-1609.
Pant, B. D., Buhtz, A., Kehr, J., and Scheible, W. R. 2008. MicroRNA399 is a long-distance signal for the regulation of plant phosphate homeostasis. Plant J. 53:731-738.

Pant, B. D., Musialak-Lange, M., Nuc, P., May, P., Buhtz, A., Kehr, J., Walther, D., and Scheible, W. R. 2009. Identification of nutrient-responsive Arabidopsis and rapeseed microRNAs by comprehensive real-time PCR profiling and small RNA sequencing. Plant Physiol. 150:1541-1555

Parniske, M. 2008. Arbuscular mycorrhiza: The mother of plant root endosymbioses. Nat. Rev. Microbiol. 6:763-775.

Paszkowski, U., Kroken, S., Roux, C., and Briggs, S. P. 2002. Rice phosphate transporters include an evolutionarily divergent gene specifically activated in arbuscular mycorrhizal symbiosis. Proc. Natl. Acad. Sci. U.S.A. 99:13324-13329.

Pearson, W. R. 2000. Flexible sequence similarity searching with the FASTA3 program package. Methods Mol. Biol. 132:185-219.

Pfaffl, M. W. 2001. A new mathematical model for relative quantification in real-time RT-PCR. Nucleic Acids Res. 29:e45.

Ramakers, C., Ruijter, J. M., Deprez, R. H., and Moorman, A. F. 2003. Assumption-free analysis of quantitative real-time polymerase chain reaction (PCR) data. Neurosci. Lett. 339:62-66.

Rausch, C., Daram, P., Brunner, S., Jansa, J., Laloi, M., Leggewie, G. Amrhein, N., and Bucher, M. 2001. A phosphate transporter expressed in arbuscule-containing cells in potato. Nature 414:462-470.

Rhoades, M. W., Reinhart, B. J., Lim, L. P., Burge, C. B., Bartel, B., and Bartel, D. P. 2002. Prediction of plant microRNA targets. Cell 110:513520.

Rubio, V., Linhares, F., Solano, R., Martin, A. C., Iglesias, J., Leyva, A., and Paz-Ares, J. 2001. A conserved MYB transcription factor involved in phosphate starvation signaling both in vascular plants and in unicellular algae. Genes Dev. 15:2122-2133.

Sanders, F. E. 1975. Effect of foliar-applied phosphate on the mycorrhizal infection of onion roots. Pages 261-276 in: Endomycorrhizas. Academic Press, London.

Scheible, W. R., Morcuende, R., Czechowski, T., Fritz, C., Osuna, D., Palacios-Rojas, N., Schindelasch, D., Thimm, O., Udvardi, M. K., and Stitt, M. 2004. Genome-wide reprogramming of primary and secondary metabolism, protein synthesis, cellular growth processes, and the regulatory infrastructure of Arabidopsis in response to nitrogen. Plant Physiol. 136:2483-2499.

Smith, S. E., and Read, D. J. 1997. Mycorrhizal Symbiosis. Academic Press. London.

Smith, S. E., Smith, F. A., and Jakobsen, I. 2003. Mycorrhizal fungi can dominate phosphate supply to plants irrespective of growth responses. Plant Physiol. 133:16-20.

Trouvelot, A., Kough, J. L., and Gianinazzi-Pearson, V. 1986. Mesure du taux de mycorhization VA d'un système radiculaire. Recherche des méthodes d'estimation ayant une signification fonctionnelle. Pages $217-$ 221 in: The Mycorrhizae: Physiological and Genetical Aspects of Mycorrhizae, INRA Press, Paris

Vance, C. P., Uhde-Stone, C., and Allan, D. L. 2003. Phosphorous acquisition and use: Critical adaptations by plants for securing a nonrenewable resource. New Phytol. 157:390-397.

Wulf, A., Manthey, K., Doll, J., Perlick, A. M., Linke, B., Bekel, T., Meyer, F., Franken, P., Küster, H., and Krajinski, F. 2003. Transcriptional changes in response to arbuscular mycorrhiza development in the model plant Medicago truncatula. Mol. Plant Microbe Interact. 16:306-314.

Zhang, Y. 2005. miRU: An automated plant miRNA target prediction server. Nucleic Acids Res. 33:W701-704.

Zhou, Z. S., Huang, S. Q., and Yang, Z. M. 2008. Bioinformatic identification and expression analysis of new microRNAs from Medicago truncatula. Biochem. Biophys. Res. Commun. 374:538-542.

Zuker, M., Jaeger, J. A., and Turner, D. H. 1991. A comparison of optimal and suboptimal RNA secondary structures predicted by free energy minimization with structures determined by phylogenetic comparison. Nucleic Acids Res. 19:2707-2714. 